\title{
MAPPING DARK MATTER NEAR GALAXY CLUSTERS
}

\author{
J.A. TYSON \\ ATET Bell Labs \\ Murray Hill, NJ 07974 USA
}

\begin{abstract}
Weak gravitational lensing can provide a direct measure of mass overdensity on scales of kpc to several Mpc. The total mass and light distribution in a survey of 32 clusters of galaxies is reviewed. The mass is derived from apodized inversion of thousands of weak lensing arclets in deep CCD shift-and-stare exposures to uniform faint surface brightness in two colors. Rest frame V band mass-to-light ratios of several hundred $h$ solar are found.
\end{abstract}

\section{Introduction}

The total mass and light distribution in deep survey data of 32 clusters of galaxies is reviewed. The mass is derived from thousands of weak lensing arclets found in deep multicolor CCD shift-and-stare exposures to uniform faint surface brightness. Masses of individual cluster galaxies may also be obtained via their additional local microlensing effects. Realistic simulations of the entire source-lens-atmosphere-detector process were performed, including multiple background galaxy redshift shells, masses for individual cluster galaxies, clumped dark matter, atmospheric seeing, and pixel sampling and sky shot noise. "Blank" field HST WFC-2 Medium Deep Survey data, together with seeing deconvolved ground-based data, are used to derive the source galaxy angular scales. Given accurate seeing and source galaxy angular scales, we find that the mass scale may be calibrated from these weak lensing data alone. Strong lensing (long arcs at the Einstein critical radius) then forms an independent check on this weak lensing mass scale calibration.

There are several source requirements for mass mapping by statistical gravitational lens inversion. Sources must (1) have redshifts large compared 


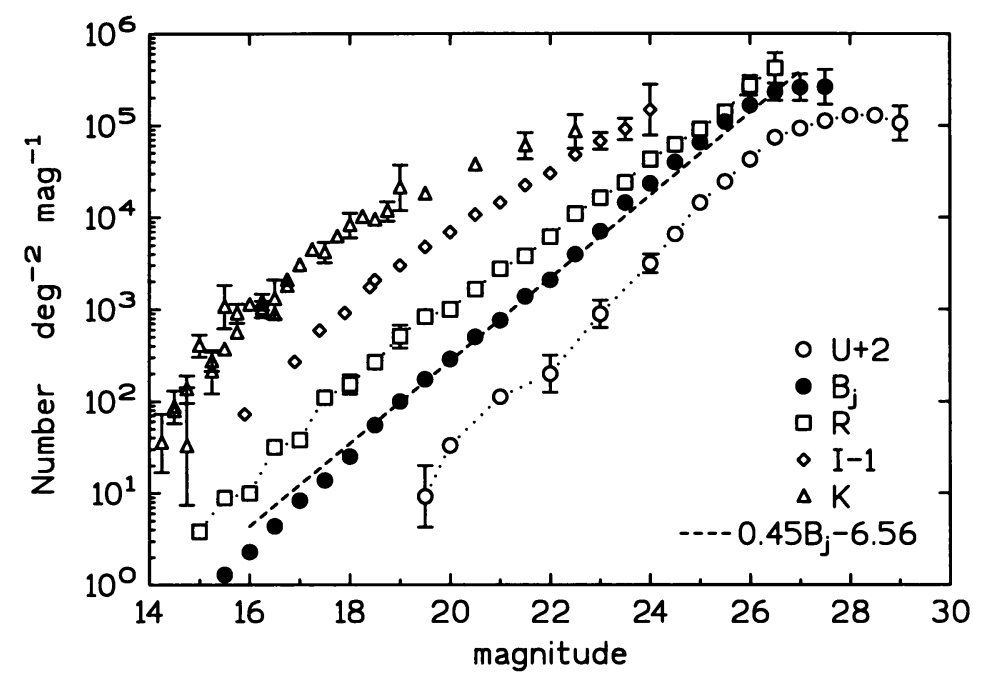

Figure 1. Faint galaxy differential number counts $\mathrm{dN}(\mathrm{m})$ as a function of magnitude in five color bands are plotted to their completeness limits. Note the decrease in slope with wavelength.

with the lens, (2) have a number density on the sky sufficient to sample the lens on relevant scales, (3) have an intrinsic angular diameter larger than the ratio of seeing FWHM to the magnification of the lens, and (4) have other properties (blue color and very low surface brightness) enabling efficient separation of the sources from lens and other foreground objects.

\section{The sources}

Figure 1 summarizes faint galaxy count data for five wavelength bands. The $\mathrm{U}$ counts are from several surveys; the $B_{j}, \mathrm{R}$, and I counts are from our most recent data on 15 fields; and the $\mathrm{K}$ counts are from Gardner et al. (1993). Number-magnitude counts which rise with magnitude like dex $(0.4 \mathrm{mag})$ or steeper continue to add to the integrated light at the faintest magnitudes, a type of Olbers' paradox. This corresponds to a number-flux $\left(N \sim S^{-n}\right)$ slope of $n=1$ near $600 \mathrm{~nm}$ wavelength. Lens-induced source number density enhancement is zero for $n=1$.

\subsection{COLORS, REDSHIFTS, AND SIZES OF FAINT GALAXIES}

While the mean $B_{j}-\mathrm{R}$ color of zero redshift galaxies is about $1.5-2$ mag at $21 B_{j}$ mag, most galaxies at 26-28 $B_{j}$ mag have $B_{j}-R<-0.2$. These extreme blue colors suggest one is seeing starburst galaxies at redshifts of $1-2$, so that the UV excess is redshifted into the $B_{j}$ passband. In a redshift- 
magnitude plot of current surveys the trend to redshift $\approx 1$ at 25 th $B_{j}$ magnitude is clear. Most of these faint galaxies appear to have redshift less than three. A typical galaxy seen at $\mathrm{z}=1$ may be a $0.1 L^{\star}$ galaxy and a survey at 25 th magnitude would cover a wide range in redshifts extending from 1-3. For arclet inversion of $z<0.3$ lenses, the lack of redshift data for these sources produces less than a $10 \%$ mass scale error.

Galaxies fainter than $26 B_{j}$ mag are found to have average exponential scale lengths of $0.2-0.5$ arcsec and typical half-light diameters of 1 arcsec. Most faint galaxies imaged at $>29 \mathrm{mag} \operatorname{arcsec}^{-2}$ surface brightness have apparent angular diameters larger than the minimum required for statistical lensing studies of foreground mass. Since angular sizes of galaxies at each magnitude show a big range, HST has been useful in resolving the small size end of this distribution (see Im et al. (1995) for a review and model comparisons). This complements the lower surface brightness studies using large ground-based telescopes.

\section{Mass density profile and maps from arclet inversion}

The mass contrast in rich clusters of galaxies distorts all the faint background galaxies within several arcminutes of the cluster. Foreground galaxy clusters at redshifts $0.2-0.5$ with radial velocity dispersions above $700 \mathrm{~km}$ $s^{-1}$ have sufficient mass density to significantly distort background galaxies of redshift greater than 0.4-1. Lensing preserves the surface brightness and spectrum of the source, so that most arcs have the very faint surface brightness and blue color of the faint blue galaxies.

To construct a rough map of the gravitational lens projected mass distribution, the distortion statistic $T_{g}(x, y)=\left(a^{2}-b^{2}\right) /\left(a^{2}+b^{2}\right)$ is computed over a grid of positions as candidate lens centers (Tyson et al. 1990). Here $\mathrm{a}(\mathrm{x}, \mathrm{y})$ and $\mathrm{b}(\mathrm{x}, \mathrm{y})$ are the $(\mathrm{r}, \theta)$ principal-axis transformed second moments of the background galaxy image (arclet) g. At any point $\vec{r}$ in the image plane we can sum over the tangential alignment of all arclets about that point, creating a continuous scalar distortion statistic

$$
D(\vec{r})=\int K(\vec{u}) T(\vec{r}-\vec{u}) d \vec{u}
$$

where the apodization kernel $K(\vec{u})$ weights source images at large radius less, and is generally of the form $K(\vec{u})=\left(u^{2}+u_{0}^{2}\right)^{-1}$ (Kaiser and Squires 1993). Our kernel also is a function of the distance to the image edges, compensating for edge effects. The distortion map $\mathrm{D}(\mathrm{x}, \mathrm{y})$ uniquely locates the lens mass, and gives its morphological shape on the sky. 


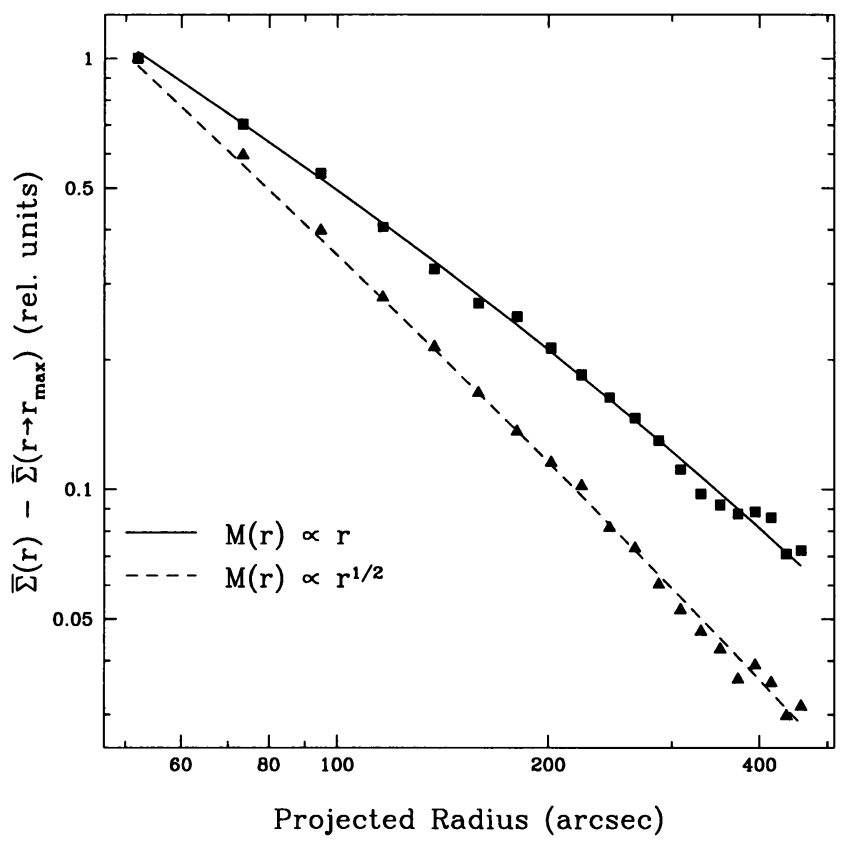

Figure 2. A radial plot of the projected mass density contrast obtained by inversion of the arclets in two $29 \mathrm{~B}$ mag arcsec ${ }^{-2}$ noisy simulations with $\sigma_{v}=1000 \mathrm{~km} \mathrm{sec}^{-1}$. The solid lines are the input mass density.

Rearranging one of Jordi Miralda's equations, the average projected mass density interior to radius $\mathrm{r}$ is given by

$$
\bar{\Sigma}(r)=\Sigma_{c} C B(r) \int_{r}^{r_{\max }} T(r) d \ln r+\bar{\Sigma}\left(r, r_{\max }\right),
$$

where $\mathrm{C}$ is the seeing and efficiency correction found in Monte Carlo tests of the data, $B(r)=\left(1-r^{2} / r_{\text {max }}^{2}\right)^{-1}, \bar{\Sigma}\left(r, r_{\max }\right)$ is the average density in the annulus between $\mathrm{r}$ and $r_{\max }$, and $\Sigma_{c}$ is the critical density. For a sufficiently large field $\bar{\Sigma}\left(r, r_{\max }\right)$ is small compared to the peak density.

Automated detection, sub-image splitting and photometry reduce the CCD images to a catalog of galaxy image properties. Faint blue galaxies have their positions, total magnitudes, and apodized second moments passed to the lens arclet inversion software. Finally, the lens projected mass overdensity (Eqn. 2) as a function of radius from the centroid of the mass distribution may be compared to the lens cluster light (baryonic) distribution. A radial plot of the projected surface mass density found in this way is shown in Figure 2 for $z_{l}=0.3, \sigma_{v}=1000 \mathrm{~km} \mathrm{sec}^{-1}$. 


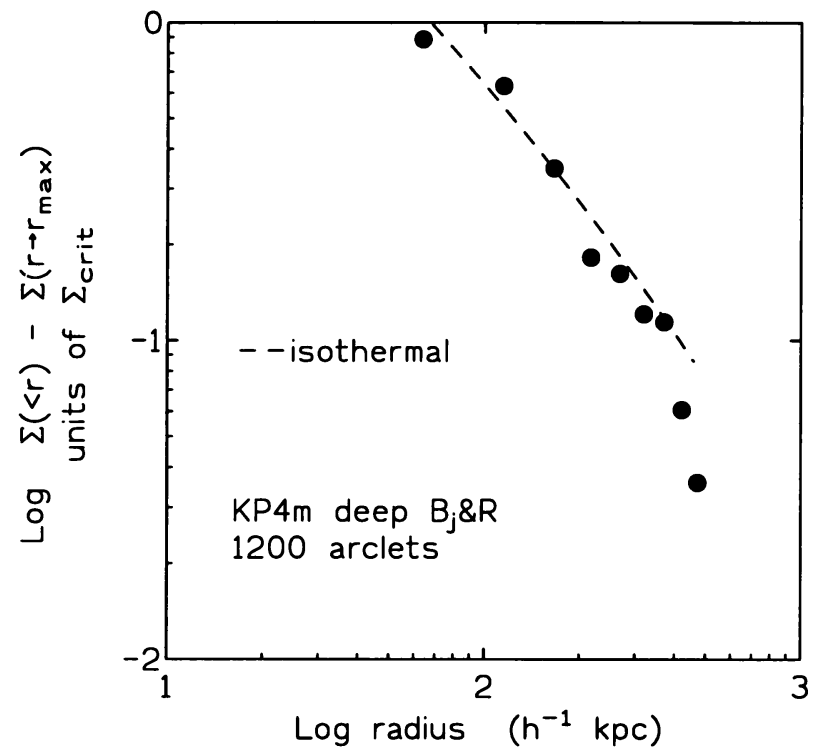

Figure 3. A radial plot of the projected mass density contrast for the $\mathrm{z}=0.39$ cluster $0024+17$. The dashed line is an isothermal profile.

\section{Results}

Selecting 50 clusters with a wide range of optical richness and X-ray luminosity, we have imaged 32 clusters over the past seven years to a uniform surface brightness limit of $29 \mathrm{~B}$ and $28 \mathrm{R}$ mag $\operatorname{arcsec}^{-2}$ : A1285, A1146, A545, A2218, A1689, A665, 2130-428, MS0839+29, A778, A2163, A732, A963, A2397, Zw3146, A2390, A1942, A2645, A1525, A1352, A528, A483, S506, MS2137-23, 2244-02, S67, 0957+561, 0024+17, 0940+46, 0303+17, 3C295, MS0451-03 and 1602+42, in order of increasing redshift, plus fifteen blank fields. Although some of these fields must be repeated with larger CCDs, several clusters are mapped beyond $1 \mathrm{~h}^{-1} \mathrm{Mpc}$ from the center. From the inversion of 6000 arclets surrounding the rich $\mathrm{z}=0.18$ cluster A1689, we find a steeper than isothermal profile (Tyson \& Fischer 1995) beyond $300 \mathrm{~h}^{-1} \mathrm{kpc}$ radius. Some clusters at high redshift are nearly as compact in mass. Figure 3 shows the mass profile for CL $0024+17$. The projected mass density contrast was obtained from 1200 arclets, excluding the bright long arcs, in the $40 \mathrm{arcmin}^{2}$ field of the 4-meter.

In rich compact clusters we find that mass traces the cluster red light, on scales $>100 h^{-1} \mathrm{kpc}$, with rest-frame $\mathrm{V}$ band mass-to-light ratios of a few hundred $h$ in solar units. The mass core radius is significantly smaller than some observed X-ray core radii in nearby clusters, suggesting that the X-ray gas may be less relaxed dynamically than the dark matter. At 
least for rich clusters these lens studies appear to confirm the large mass which was implied by virial calculations using velocity dispersions. For the optically compact clusters we find the dark matter peak densities are about $10^{5}$ times the critical density for closure, with core radii up to $70 \mathrm{kpc}$. The relation of the faint diffuse optical nebulosity near the cores of many of these clusters to the dark matter or X-ray is intriguing, and merits further study; it is not correlated with either bright galaxies or the mass map.

The scale of the sub-clumping of the dark matter within clusters of galaxies is also interesting. The dark matter cannot all be clumped on galaxy mass scales, otherwise the long arcs seen in several relaxed cluster lenses would be broken up on several arcsecond scales and arcs or distorted "ringlets" would form around cluster galaxies. These appear in simulations where masses in excess of the Faber-Jackson mass or non-truncated isothermal mass distributions are assigned to cluster galaxies, but are not seen in the observations. On scales $<100 \mathrm{kpc}$, dark matter does not clump with individual cluster galaxies other than the central cD galaxy. Most cluster galaxies appear to be no more massive than field galaxies, so the dark matter in clusters is primarily in the diffuse component. We have found several cases of multiple mass clumps on $300 \mathrm{~h}^{-1} \mathrm{kpc}$ scales.

Ultimately, comparing the mass morphologies found near clusters with high resolution $\mathrm{N}$-body simulations for various cosmogonies will further narrow the candidates for dark matter. Weak gravitational lensing arclet inversion provides a direct measure of mass overdensity on scales of $\mathrm{kpc}$ to several Mpc. Larger scale applications of this dark matter mapping may eventually find clumped dark matter unrelated to galaxies or clusters of galaxies. Mosaics of CCDs make such a large scale search for coherent alignment in the distant faint galaxies particularly attractive, and dark matter on angular scales up to degrees can in principle be studied in this way. Preliminary measurements of the arclet orientation correlation function in random fields are below the CDM prediction.

Acknowledgements: My collaborators in this research are Gary Bernstein, Phil Fischer, Raja Guhathakurta, George Rhee, Jordi Miralda-Escudé, Ed Turner, Wes Colley and Rick Wenk.

\section{References}

Gardner, J.P., Cowie, L.L., \& Wainscoat, R.J., 1993, ApJL, 415, L9

Im, M., Casertano, S., Griffiths, R.E., Ratnatunga, K.U. \& Tyson, J.A., 1995, ApJ, 441, 494

Kaiser, N. \& Squires, G., 1993, ApJ, 404, 441

Tyson, J.A. \& Fischer, P., 1995, ApJ, 96, 1

Tyson, J.A., Valdes, F., \& Wenk, R.A., 1990, ApJL, 349, L1 